\title{
An Update on the Current Role of High Resolution Anoscopy in Patients With Anal Dysplasia
}

\author{
ANURAGH GUDUR ${ }^{1 *}$, DEEPAK SHANMUGANANDAMURTHY ${ }^{*}$, ZSOFIA SZEP $^{2}$ and JUAN LUCAS POGGIO ${ }^{3}$ \\ ${ }^{1}$ Drexel University College of Medicine, Philadelphia, PA, U.S.A.; \\ ${ }^{2}$ Division of Infectious Disease, Department of Medicine, Drexel University, Philadelphia, PA, U.S.A.; \\ ${ }^{3}$ Division of Colorectal Surgery, Department of Surgery, Drexel University, Philadelphia, PA, U.S.A.
}

\begin{abstract}
The incidence of anal squamous cell carcinoma (ASCC) in the U.S. is increasing, particularly in immunocompromised patients. Preventative screening is used to monitor precancerous dysplasia known as anal intraepithelial neoplasia (AIN), which can progress to ASCC. High Resolution Anoscopy (HRA) is one screening procedure that uses a colposcope with acetic acid and Lugol's iodine solution to visualize the anal epithelium for AIN. HRA has offered potential in managing AIN, but as more evidence emerges it is unclear whether HRA provides definitive benefit. This narrative review updates readers on the efficacy of HRA in screening AIN, compares HRA to other techniques, compares the use of HRA in various high-risk populations, and offers practical information on the use of HRA. Keyword searches were conducted using MEDLINE, Web of Science, and Cochrane Library. Current literature is mixed regarding the utility of HRA. Further randomized controlled trials are needed to definitively assess the role of HRA.
\end{abstract}

The incidence of anal squamous cell carcinoma (ASCC) is rising quickly (1). In the United States alone, the incidence has doubled within the last three decades (2). High-risk populations - such as human immunodeficiency virus (HIV) patients, human papilloma virus (HPV)-infected patients,

*The two Authors contributed equally to this study.

Correspondence to: Juan Lucas Poggio, MD, MS, FACS, FASCRS, Associate Professor of Surgery, Chief, Division of Colorectal Surgery, Department of Surgery, Drexel University College of Medicine and Hahnemann University Hospital, 245 N. 15th Street, MS 413, Philadelphia, PA 19102-1192, U.S.A. Tel: +1 2157621750, Fax: +1 2157628389, e-mail: jlp54@drexel.edu

Key Words: Anal squamous cell carcinoma, anal intraepithelial neoplasia, high resolution anoscopy, anal dysplasia, review. men who have sex with men (MSM), and transplant recipients - are especially affected by ASCC (1). In the general population the incidence is 2 per 100,000 and as high as 131 per 100,000 among HIV-infected MSM (3). By comparison, the incidence of lung cancer - the leading cause of cancer death - is 62 per 100,000 (4).

ASCC is often preceded by precancerous abnormalities in the anal epithelium known as anal intraepithelial neoplasia (AIN). AIN is further divided into three subcategories (Grades 1,2 , and 3 ) based on the severity of the condition. Low-grade AIN (LGAIN), also known as low-grade squamous intraepithelial lesions (LSIL), refers to Grade 1 disease. It is typically classified as mild dysplasia. High-grade AIN (HGAIN), otherwise known as high-grade squamous intraepithelial lesions (HSIL), usually accounts for Grade 2 and Grade 3 disease. It is characterized as moderate to severe dysplasia, and sometimes even anal carcinoma in situ (5).

Screening has been recommended as a method to monitor AIN and prevent ASCC (6). Current screening modalities used include anal cytology (anal pap smear), standard anoscopy, or high resolution anoscopy (HRA) $(1,7)$. HRA is a relatively new procedure that utilizes a colposcope or microscope with acetic acid and Lugol's iodine solution to carefully inspect the anal epithelium for abnormal changes (5). HRA has been endorsed as a promising prospect with strong potential in managing AIN, but there is a growing body of evidence that may suggest otherwise (7-11). Moreover, formal training in HRA only began in 2005, so only a small proportion of clinicians are sufficiently trained in performing the procedure $(12,13)$. There is still some lack of awareness in the medical community over the utility of HRA (14). On a cost-per-use basis, HRA is also a very expensive procedure (15). In addition to these factors, the lack of consistent and well-supported guidelines on the use of HRA make it even more challenging for clinicians to incorporate the procedure into their practice (2). 
Given the rising incidence of ASCC, particularly in highrisk populations, it is important to focus on the utility of HRA as a screening, surveillance, and diagnostic tool. Based on currently available literature, the objective of this review is to examine the efficacy of HRA in screening AIN, compare HRA to other available techniques, compare the use of HRA in various high-risk populations, and offer practical information about using HRA.

\section{Methods}

The methodological approach of this paper was to conduct a narrative literature review based on the best practice recommendations (16). A literature search was conducted in May 2018 using the MEDLINE (PubMed), Web of Science, and Cochrane Library databases. The search used the keywords "anoscopy", "high resolution anoscopy", "anal dysplasia", "anal intraepithelial neoplasia", "anal intraepithelial dysplasia", "anal squamous cell carcinoma", "anal cancer", "anal pap smear", "anal cytology", "ASCC", "AIN", "HGAIN", "LGAIN", and "HRA". Papers published between 2000 and 2018 that contained the keywords in the title or abstract were selected for review. The inclusion criteria were studies relating only to high resolution anoscopy, but encompassing all types of studies, patient populations, languages, and journals. Exclusion criteria were articles without full text available or that did not discuss high resolution anoscopy. The potential articles were reviewed to assess relevance to the topic. Irrelevant or duplicate studies were excluded. Additional articles from reference lists of studies were also included. A flow chart of the literature search is shown in Figure 1.

\section{Discussion}

Efficacy of HRA in detecting AIN. Current literature illustrates the efficacy of HRA in detecting HGAIN, especially when tested in high-risk populations. Interestingly, national screening guidelines for anal cancer are non-existent as noted by Santorelli et al. in their retrospective study of a five-year experience in screening for ASCC (17). They noted, however, that the most popular screening algorithm supported by researchers utilizes HRA to analyze any atypical cytology results and to conduct annual follow-up screening for AIN. When studying the efficacy of HRA on a group of 300 HIVinfected MSM, Sendagorta et al. demonstrated that HRA has a high positive predictive value (PPV) of detecting HSIL (79.8\%) (9). In addition, Berry et al. concluded that utilizing HRAguided biopsy to assess anal lesions in MSM is an effective tool for diagnosing HGAIN (18). The authors further suggested that HRA should be the standard for detecting HGAIN, as most HGAIN is not palpable by digital rectal examination or simple anoscopy except in the case of advanced lesions. An Australian study by Roberts et al. on the histological outcomes of highgrade cytopredictions with HRA in MSM also complemented this finding (19). Data showed that high-grade cytopredictions are meaningful with a histological HSIL confirmed in more than $90 \%$ of cases after 3 HRAs over a 12-month period.
Despite the promising results of HRA in detecting HGAIN, there are some setbacks that a multitude of studies have pointed out. Sendagorta et al. noted that although HRA has a very high PPV in predicting lesions of any type on biopsy (93\%), there is a significantly lower PPV for detecting HSIL specifically $(65.5 \%)$ (9). This is a weakness of HRA that diminishes its use in screening for undetected HSIL. Moreover, the addition of random biopsy to HRA significantly increases both the number of HSILs and participants with HSIL identified. In their study, Silvera et al. showed that without the addition of random biopsy of anal quadrants without apparent HSIL, $12.7 \%$ of HSILs $(n=33)$ and $9.8 \%$ of participants with HSIL $(n=13)$ would have gone unidentified (10). Furthermore, studies delving into the results of HRA in detecting AIN have indicated that despite its high sensitivity (90\%), HRA has a specificity of only $19.23 \%$ for the diagnosis of anal squamous intraepithelial lesions (ASIL) (11). Gimenez et al. elaborated on this statistic, citing that these rates vary greatly $(59-100 \%$ sensitivity; 66-74\% specificity) and postulated that it is due to the subjectivity of HRA and the fact that it is a user-, experience-, and equipment-dependent test.

HRA has a strong ability to detect HSIL that can be augmented by other screening and diagnostic techniques. The epidemiological studies of HSIL may underestimate the prevalence of HSIL if the results of anal cytology and HRA are not combined. Machalek et al. showed that the diagnosis of ASIL is hindered by the limited sensitivity of both anal cytology sampling and HRA used alone, especially in the context of LSIL or missed HSIL (20). The authors demonstrated that combining the results of anal cytology and HRA leads to the diagnosis of more biologically relevant disease. Nahas et al. arrived at a similar conclusion that HRA should be used in conjunction with anal cytology, as anal cytology by itself is not enough to make a confident diagnosis of ASIL (21). A study in Portugal by Silva et al. supported this notion of using HRA in combination with another technique such as anal cytology by noting that there was poor correlation between anal cytology, histopathology, and anoscopic visual impression alone (kappa-agreement $=$ $0.20)$ (1). In addition to anal cytology, random biopsy of anal quadrants non-suggestive of HSIL is another potential technique that can be used in conjunction with HRA to increase the number of HSILs identified per person as well as the number of persons with HSIL (10).

Comparison of HRA to other techniques. Various techniques are currently employed in the screening and management of anal dysplasia. It is helpful to compare the efficacy of HRA to other techniques, as several studies have been performed in recent years.

Many primary care providers perform anal pap smears as part of routine care in high-risk individuals (21). When 


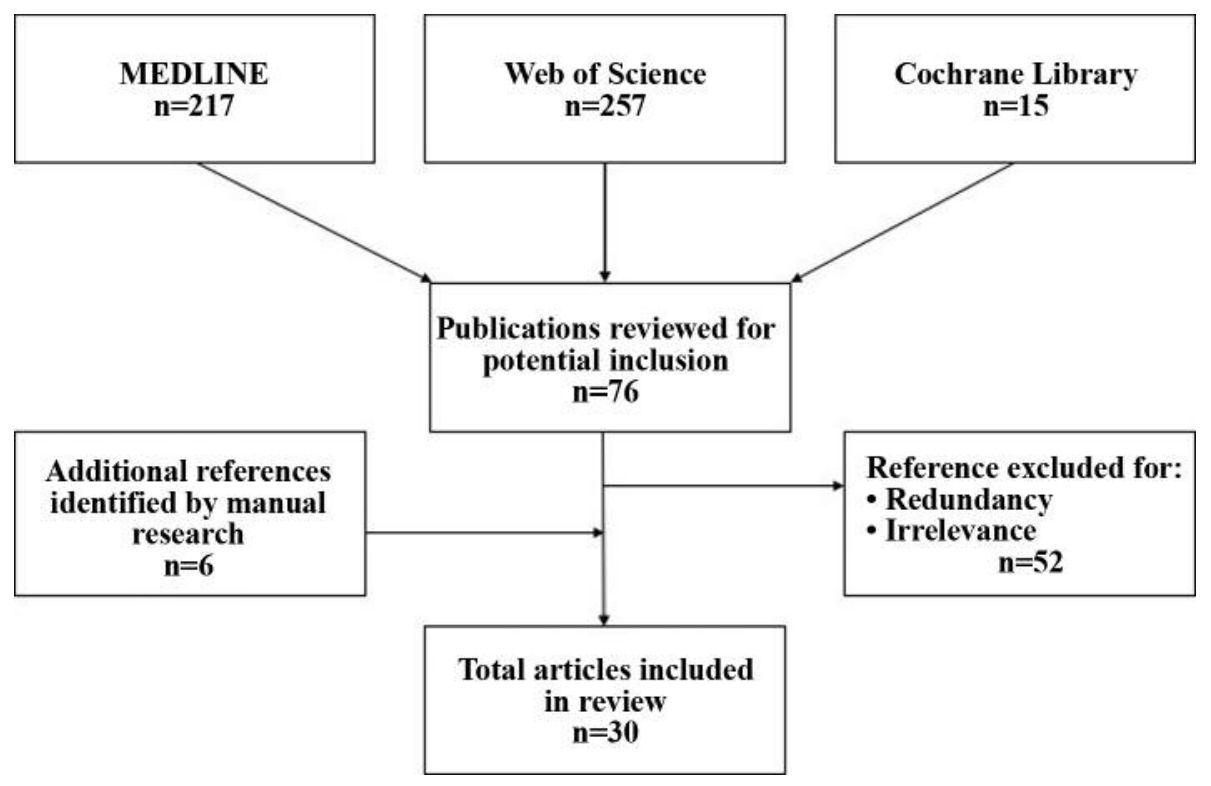

Figure 1. Flow chart of literature search.

compared to the anal pap smear, HRA has advantages and disadvantages as a screening tool. HRA is beneficial because it is more sensitive: Nahas et al. demonstrated that the anal pap smear has a sensitivity of $60 \%$, which was well below the sensitivity of HRA $(90 \%)$ as reported by Gimenez et al. (11, 21). The high sensitivity of HRA allows physicians to rule out dysplasia. In fact, Nahas recommended that anal pap smear alone is not enough to be confident, and that HRA should be added to screening. However, HRA is less specific than the anal pap smear, which has a specificity of $60 \%(11,21)$. The low specificity could create a high number of false positives.

Some studies have demonstrated that HRA was not different or comparable to other procedures such as standard anoscopy or anal chromoendoscopy (ACE). Crawshaw et al., for example, retrospectively compared HRA versus standard anoscopy in 424 patients with squamous-cell dysplasia (7). Only 3 patients progressed to anal cancer in both groups combined. Using a 5-year anal cancer progression rate as a measure of performance, the HRA group was not significantly different from the standard anoscopy group. HRA patients had $4.5 \%$ anal cancer progression rate while standard anoscopy patients had a $6 \%$ progression rate $(p=0.37)$. The authors, however, stated that their study lacked sufficient power to significantly distinguish small differences in anal cancer progression rates between groups. A different prospective cohort study by Oette et al. compared ACE versus HRA in identifying AIN in 211 high-risk patients (22). ACE applies topical color dyes to inspect any epithelial abnormalities using an endoscope. ACE demonstrated a sensitivity of $85 \%$, specificity of $55 \%$, PPV of $50 \%$, and negative predictive value (NPV) of $87 \%$. Oette compared these values to HRA using data from a previously published study (sensitivity of HRA was $90 \%$, specificity was $19 \%$, PPV was $42 \%$, and NPV was $75 \%$ ) and concluded that ACE was similar to HRA in detecting anal dysplasia (11).

In contrast, Pineda et al. found that HRA is a superior method in the management of HSIL (23). The retrospective study of 246 patients concluded that HRA-targeted surgical treatment proved to be a successful method of managing HSIL, with a cancer progression rate of 1.2 percent over a mean follow-up period of 41 months. This rate was far lower than that of expectant management, which had a cancer progression rate of 8.5 to $13 \%$.

Comparison of use of HRA in various high-risk patient populations. Given the nature of anal cancer and the specific high-risk populations that it primarily afflicts, numerous studies have discussed the use of HRA in these specific cohorts. The chief high-risk populations susceptible to anal cancer in which HRA has been tested include patients with Bowen's disease (AIN III or anal carcinoma in situ), those with high-risk types of HPV, men who have sex with men (MSM), individuals living with HIV, and renal graft recipients.

In the setting of patients with Bowen's disease (AIN III or anal carcinoma in situ), Chung \& Rosenfeld have delved into the use of HRA in the diagnosis and treatment of this malady in a private practice setting (24). A key finding in their study which included patients that were both HIV-positive and HIV- 
negative is that HRA allowed both identification and treatment of more extensive cases of Bowen's disease compared to wide excision. These results demonstrated a strikingly different AIN recurrence rate between the subpopulations, $25 \%(n=1)$ in HIVnegative patients and $65 \%(n=11)$ in HIV-positive patients. However, the sample size was quite small in this private practice study and these rates vary greatly among other studies. Chang et al. performed a similar study on patients with HGAIN noting that they were able to eradicate disease in HIVnegative patients ( 0 of 8 patients with recurrence) and had a $79 \%$ recurrence rate ( 23 of 29 patients with recurrence) in the HIV-positive subgroup (25). Nonetheless, the authors conclude "HRA is a safe and effective means of following patients with AIN III for recurrence, even in the community setting."

MSM constitute a large population among those with high-grade AIN and thus play a crucial role in understanding the efficacy of HRA in the prevention of anal cancer. Of the studies done on HIV-positive MSM, high levels of HGAIN were identified in this cohort and HRA was found to have a high PPV $(10,25)$. In one longitudinal pilot study of HRA screening of HIV-positive MSM, Dalla Pria et al. found that the incidence of anal cancer after an HRA screening program was 2.7 per 1,000 person-years (26). However, this study did not use a control group due to ethical concerns, so the results could not be placed into context. This made it difficult to assess the significance of HRA screening in MSM.

In addition to being in the high-risk population of MSM living with HIV, Berry et al. illustrated that when patients had at least one high-risk type of HPV present (types 16, 18, $31,33,35,39,45,51,52,56,58,59$, or 68$)$, the sensitivity and specificity of HRA to detect HGAIN were altered (18). When analyzing the study group of 23 MSM living with HIV that had at least one high-risk type of HPV, sensitivity decreased slightly from $93 \%$ (range $=68-100 \%$ ) to $87 \%$ (range $=60-98 \%$ ) whereas specificity markedly increased from $12 \%$ (range $=1-36 \%$ ) to $41 \%$ (range $=18-67 \%)$. It is important to note that HPV status of the patient can further alter HRA findings with arguably better test results coming from those with a high-risk type of HPV.

Immunocompromised patients regardless of sexual history and HPV-status are at risk for developing anal cancer and are generally comprised of transplant recipients and individuals living with HIV. The literature has widely shown that anal cytology has good ability in terms of its sensitivity, but when compared to the HRA-guided biopsy, it underestimates the grade of dysplasia in individuals with HIV and renal graft recipients $(11,21,27,28)$. Thus, HRA is suggested as a means to verify and rule out the presence of AIN in these populations. Betancourt et al. performed a study that used HRA-guided biopsy for cases of negative cytology and found a false-negative rate of $7 \%(n=6)(27)$. There was visible pathology on HRA in these patients that led to a diagnosis that would have otherwise gone undetected with anal cytology alone. Nahas et al. also discussed this in their study of screening individuals with HIV for anal dysplasia and recommend that anal cytology and HRA be used in conjunction for better diagnostic confidence and to ensure that these populations are screened thoroughly (21).

Immunocompetent, HIV-negative patients with HSIL with the risk of progression to anal cancer can also benefit greatly with HRA-guided excision treatment. The traditional approach for these patients involves punch biopsy mapping of the anus performed in the operating room with frozen section analysis followed by wide local excision (29). Despite the lack of literature on immunocompetent patients, it is possible to get a glimpse of how HRA performs in this cohort. A study by Pineda et al. showcases the results on HRA in the planned treatment of AIN in HIV-negative patients. The authors stated that their observed rate of HSIL progression to cancer with [their] approach of treatment, surveillance, and re-treatment compares favorably to that reported by others $(2.4 \%$ versus $5.7-13 \%$ ) (30). Although the initial recurrence rate of lesions was high following HRA-guided procedures, these recurrent lesions were well-controlled by further HRA-guided treatments, even in severe cases.

Practical information for clinicians interested in using HRA. Given the relative novelty of HRA, many physicians are unfamiliar with the procedure and may need more information regarding the practical aspects of utilizing HRA $(30,31)$. In fact, Berry-Lawhorn et al. noted that only $46 \%$ of clinicians who performed HRA received any formal training in the procedure (12). It is helpful to understand the important practical features of HRA, such as the technical training required, pain associated with the procedure, and cost-effectiveness.

Studies demonstrated that physicians require a significant amount of time to achieve competency in utilizing HRA. Nahas et al. emphasized the notion that proficiency in performing HRA requires direct practice over a span of years (21). Swedish et al. offered further support to this idea by demonstrating an increase in the ability to identify anal dysplasia by $16 \%$ after 10 years of experience with HRA (32). Hillman et al. also examined the effect of experience on the ability of an anoscopist to detect lesions (33). The study showed a definitive improvement in the ability to detect dysplasia after two years of experience, most likely due to an increase in technical expertise. Hillman et al. even went on to suggest a minimum of 500 procedures to attain the greatest diagnostic ability in performing HRA. A separate study by Machalek et al. noted that physicians may achieve maximum diagnostic yield after 200 procedures, which would also take a significant amount of time (20).

Evidence from the literature appears to demonstrate that HRA is a difficult procedure with a long learning curve. Unique anatomical features of the anal region such as 
mucosal folds or the presence of hemorrhoids can make HRA even more challenging to master (2).

Postoperative pain in patients has been cited as another concern over performing HRA (24). Pineda et al. reports that the pain is similar to that of a hemorrhoidectomy but can be managed with analgesics (23). However, other studies have differed from this viewpoint, citing that HRA has minimal pain associated with the procedure. Repiso-Jiménez et al. concluded that HRA is "painless and well-tolerated" (34). This conclusion was further validated by De-Masi et al., who conducted a study to analyze the acceptability of HRA in 380 patients who underwent the procedure. Patients reported a median pain score of 2 on a scale of 0 to 10 ( 0 indicated no pain. 10 indicated severe pain) (35). The results also demonstrated that $98 \%$ of women and $99 \%$ of men were willing to undergo an HRA examination in the future. As such, it appears that the pain associated with HRA is not truly problematic, with treatments available to help mitigate this concern in patients.

Finally, in terms of a cost-benefit analysis, HRA has proven to be the most cost-effective screening modality in a study by Lam et al. (15). Although cytology testing via anal Pap smear had a lower cost per procedure than HRA, the low specificity of cytology testing led to a high number of false positives, which diminished the advantage of lower cost. The higher cost of HRA was justified by its sensitivity, making it cost-effective. A separate study by Assaoumau et al. also concluded that HRA is a cost-effective screening strategy (36). According to Assaoumau, using repeat HRA (HRA at 6 months + HRA at 12 months) as a screening strategy was more cost-effective than using HRA as a secondary procedure merely to confirm abnormal cytology results.

Looking toward the future: The ANCHOR study and its potential implications for HRA. More research is needed to attain meaningful conclusions regarding HRA. A large-scale randomized trial, the Anal Cancer HSIL/Outcomes Research study, is currently recruiting patients with HSIL to determine whether treating HSIL leads to lower anal cancer progression rates than active monitoring alone (37). This landmark trial will help determine the best strategy for preventing anal cancer (treatment versus observation). However, this study will not directly test HRA. All participants will undergo HRA to identify HSIL and determine their eligibility. The study is not intended to elucidate whether HRA is better than other techniques such as standard anoscopy. Therefore, questions will still remain regarding the efficacy of HRA and whether it is superior to other techniques.

\section{Conclusion}

Current literature appears to be mixed on the use of HRA. HRA has proven to be a low-pain, cost-effective method to detect high-grade lesions. It has also been shown to be more sensitive than the anal pap smear, one of the most popular screening tests for AIN. Studies further show that in order to be thorough in screening for AIN, anal cytology should be used in conjunction with HRA as there is a notable falsenegative rate for simply performing the anal pap smear. However, there are concerns that HRA was subpar in effectively and consistently detecting low-grade lesions. Furthermore, without the addition of random biopsy to HRA, a significant proportion of HSIL and participants with HSIL would have gone unidentified $(12.7 \%$ and $9.8 \%$ respectively). Certain studies have also pointed out that HRA was no better than other screening methods, such as ACE or standard anoscopy. This elicits curiosity about the true usefulness of HRA in detecting AIN.

With respect to high-risk populations, current literature appears to show that HRA is an effective and safe method in monitoring patients with AIN, especially AIN III, for recurrence and progression to cancer. This seems to hold true in both the academic and community setting, but further studies are needed in the community setting to fully support this result. For screening MSM, the presence of a high-risk strain of HPV improves HRA specificity greatly while marginally decreasing sensitivity. In the immunocompetent patient group, HRA was able to manage lesions even in patients with more serious anal dysplasias, despite facing a higher initial recurrence rate. In addition, the observed rate of HGAIN progression to cancer with HRA treatment was considerably lower than that of the standard punch biopsy treatment $(2.4 \%$ vs. $5.7-13 \%)$. Unfortunately, there is a dearth of studies focusing on the immunocompetent population to further substantiate these promising claims.

On that note, further research must be conducted to establish conclusive evidence. The studies used in this paper did not include any randomized controlled trials and often used heterogenous patient groups. Plus, the fact that HRA itself is a subjective, user- and experience-dependent test with many physicians performing the procedure without formal training limits the ability to offer a definitive verdict on HRA. There is a need for defined, large-scale randomized controlled trials to clarify the effectiveness of HRA in detecting AIN and also determine whether it is superior to standard anoscopy. The ongoing ANCHOR study is not specifically intended to test the performance of HRA or compare it to other techniques. Once HRA can truly demonstrate its value, it is recommended that clinicians take into consideration the amount of experience and skill needed to gain proficiency in the procedure. HRA has a steep learning curve, so it is important to receive proper training to resolve the current shortage of trained physicians.

In conclusion, HRA has shown promise, but further studies are required as some questions remain about whether HRA is truly effective and definitively superior to other techniques, 
particularly in high risk populations. Given the current lack of consistent and well-supported guidelines, more evidence is needed to clearly define the role of HRA in clinical practice.

\section{References}

1 Silva M, Peixoto A, Sarmento JA, Coelho R and Macedo G: Anal cytology, histopathology and anoscopy in an anal dysplasia screening program: is anal cytology enough? Rev Esp Enferm Dig 110: 109-114, 2018.

2 Brady JT, Ko B and Stein SL: High-resolution anoscopy: Is it necessary in the management of anal epithelial neoplasia. Semin Colon Rectal Surg 28: 102-105, 2017.

3 Silverberg MJ, Lau B, Justice AC, Engels E, Gill MJ, Goedert JJ, Kirk GD, D'Souza G, Bosch RJ, Brooks JT, Napravnik S, Hessol NA, Jacobson LP, Kitahata MM, Klein MB, Moore RD, Rodriguez B, Rourke SB, Saag MS, Sterling TR, Gebo KA, Press N, Martin JN and Dubrow R: Risk of anal cancer in HIVinfected and HIV-uninfected individuals in North America. Clin Infect Dis 54: 1026-1034, 2012.

4 Dela Cruz CS, Tanoue LT and Matthay RA: Lung Cancer: Epidemiology, Etiology, and Prevention. Clin Chest Med 32: 605-644, 2011.

5 Jay N: High-resolution anoscopy: Procedure and findings. Sem Colon Rectal Surg 28: 75-80, 2017.

6 Wasserman P, Rubin DS and Turett G: Review: Anal intraepithelial neoplasia in HIV-infected men who have sex with men: Is screening and treatment justified? AIDS Patient Care STDS 31: 245-253, 2017.

7 Crawshaw BP, Russ AJ, Stein SL, Reynolds HL, Marderstein EL, Delaney CP and Champagne BJ: High-resolution anoscopy or expectant management for anal intraepithelial neoplasia for the prevention of anal cancer. Dis Colon Rectum 58: 53-59, 2015.

8 Welton ML, Oberhelman HA and Lightner AL: Incorporating anal dysplasia screening and management into a surgical practice. Semin Colon Rectal Surg 28: 111-113, 2017.

9 Sendagorta E, Herranz P, Guadalajara H, Bernardino JI, Viguer JM, Beato MJ, García-Olmo D and Peña JM: Prevalence of abnormal anal cytology and high-grade squamous intraepithelial lesions among a cohort of HIV-infected men who have sex with men. Dis Colon Rectum 57: 475-481, 2014.

10 Silvera R, Gaisa MM and Goldstone SE: Random biopsy during high-resolution anoscopy increases diagnosis of anal high-grade squamous intraepithelial lesions. J Acquir Immune Defic Syndr 65: 65-71, 2014.

11 Gimenez F, Costa-E-Silva ITD, Daumas A, Araújo JD, Medeiros SG and Ferreira L: The value of high-resolution anoscopy in the diagnosis of anal cancer precursor lesions in HIV-positive patients. Arq Gastroenterol 48: 136-145, 2011.

12 Berry-Lawhorn JM, Chrobak D, Jay N, and Palefsky JM: Who is ready to screen for anal squamous intraepithelial lesions and why should they perform high-resolution anoscopy? Sex Transm Dis 41: 254-256, 2014.

13 Smyczek P, Singh AE and Romanowski B: Anal intraepithelial neoplasia: review and recommendations for screening and management. Int J STD AIDS 24: 843-851, 2013.

14 Goon P, Morrison V, Fearnhead N, Davies J, Wilson C, Jephcott $\mathrm{C}$, Sterling $\mathrm{J}$ and Crawford R: High resolution anoscopy may be useful in achieving reductions in anal cancer local disease failure rates. Eur J Cancer Care 24: 411-416, 2015.

15 Lam JM, Hoch JS, Tinmouth J, Sano M, Raboud J and Salit IE: Cost-effectiveness of screening for anal precancers in HIVpositive men. AIDS 25: 635-642, 2011.

16 Ferrari R: Writing narrative style literature reviews. Medical Writing 24: 230-235, 2015.

17 Santorelli C, Leo CA, Hodgkinson JD, Baldelli F, Cantarella F and Cavazzoni E: Screening for squamous cell anal cancer in HIV positive patients: A Five-Year Experience. J Invest Surg 17, 2017. doi: 10.1080/08941939.2017.1334845. [Epub ahead of print]

18 Berry JM, Palefsky JM, Jay N, Cheng SC, Darragh TM and Chin-Hong PV: Performance characteristics of anal cytology and human papillomavirus testing in patients with high-resolution anoscopy-guided biopsy of high-grade anal intraepithelial neoplasia. Dis Colon Rectum 52: 239-247, 2009.

19 Roberts JM, Jin F, Poynten IM, Law C, Templeton DJ, Thurloe JK, Garland SM, Grulich AE, Farnsworth A and Hillman RJ: Histological outcomes of anal high-grade cytopredictions. Cancer Cytopathol 126: 136-144, 2018.

20 Machalek DA, Poynten IM, Jin F, Hillman RJ, Templeton DJ, Law C, Roberts JM, Tabrizi SN, Garland SM, Farnsworth A, Fairley CK and Grulich AE: A composite cytology-histology endpoint allows a more accurate estimate of anal high-grade squamous intraepithelial lesion prevalence. Cancer Epidemiol Biomarkers Prev 25: 1134-1143, 2016.

21 Nahas CS, da Silva Filho EV, Segurado AA, Genevcius RF, Gerhard R, Gutierrez EB, Marques CF, Cecconello I and Nahas SC: Screening anal dysplasia in HIV-infected patients: is there an agreement between anal pap smear and high-resolution anoscopyguided biopsy? Dis Colon Rectum 52: 1854-1860, 2009.

22 Oette M, Wieland U, Schunemann M, Haes J, Reuter S, Jensen BE, Sagir A, Pfister $\mathrm{H}$ and Häussinger D: Anal chromoendoscopy using gastroenterological video-endoscopes: A new method to perform high resolution anoscopy for diagnosing intraepithelial neoplasia and anal carcinoma in HIVinfected patients. Z Gastroenterol 55: 23-31, 2017.

23 Pineda CE, Berry JM, Jay N, Palefsky JM and Welton ML: High-resolution anoscopy targeted surgical destruction of anal high-grade squamous intraepithelial lesions: a ten-year experience. Dis Colon Rectum 51: 829-835, 2008.

24 Chung AP and Rosenfeld DB: Intraoperative high-resolution anoscopy: a minimally invasive approach in the treatment of patients with Bowen's disease and results in a private practice setting. Am Surg 73: 1279-1283, 2007.

25 Chang GJ, Berry JM, Jay N, Palefsky JM and Welton ML: Surgical treatment of high-grade anal squamous intraepithelial lesions. Dis Colon Rectum 45: 453-458, 2002

26 Dalla Pria A, Alfa-Wali M, Fox P, Holmes P, Weir J, Francis N and Bower M: High-resolution anoscopy screening of HIVpositive MSM: longitudinal results from a pilot study. AIDS 28 : 861-867, 2014.

27 Betancourt EM, Wahbah MM, Been LC, Chiao EY, Citron DR and Laucirica R: Anal cytology as a predictor of anal intraepithelial neoplasia in HIV-positive men and women. Diagn Cytopathol 41: 697-702, 2013.

28 Tramujas da Costa e Silva I, de Lima Ferreira LC, Santos Gimenez F, Gonçalves Guimarães RA, Botinelly Fujimoto L, Barbosa Cabral CR, Venturim Mozzer R and de Souza Atala L: 
High-resolution anoscopy in the diagnosis of anal cancer precursor lesions in renal graft recipients. Ann Surg Oncol 15: 1470-1475, 2008.

29 Margenthaler JA, Dietz DW, Mutch MG, Birnbaum EH, Kodner IJ and Fleshman JW: Outcomes, risk of other malignancies, a need for formal mapping procedures in patients with perianal Bowen's disease. Dis Colon Rectum 47: 1655-1660, 2004.

30 Pineda CE, Berry JM, Jay N, Palefsky JM and Welton ML: High resolution anoscopy in the planned staged treatment of anal squamous intraepithelial lesions in HIV-negative patients. J Gastrointest Surg 11: 1410-1415, 2007.

31 Albuquerque A: High-resolution anoscopy: Unchartered territory for gastroenterologists? World J Gastrointest Endosc 7: 10831087, 2015.

32 Swedish KA, Lee EQ and Goldstone SE: The changing picture of high-grade anal intraepithelial neoplasia in men who have sex with men: The effects of 10 years of experience performing high-resolution anoscopy. Dis Colon Rectum 54: 1003-1007, 2011.

33 Hillman RJ, Gunathilake MP, Jin F, Tong W, Field A and Carr A: Ability to detect high-grade squamous anal intraepithelial lesions at high resolution anoscopy improves over time. Sex Health 13: 177-181, 2016.
34 Repiso-Jiménez JB, Padilla-Espana L, Fernández-Morano T and de Troya-Martín M: Screening for anal intraepithelial neoplasia: high-resolution anoscopy-guided biopsy of the anal canal. Actas Dermosifiliogr 108: 65-66, 2017.

35 De-Masi A, Davis E, Cuming T, Chindawi N, Pesola F, Cappello C, Chambers S, Bowring J, Rosenthal AN, Sasieni P and Nathan M: The acceptability of high resolution anoscopy examination in patients attending a tertiary referral centre. BMC Cancer 18: $554,2018$.

36 Assoumou SA, Mayer KH, Panther L, Linas BP and Kim JJ: Cost-effectiveness of surveillance strategies after treatment for high-grade anal dysplasia in high risk patients. Sexually transmitted diseases 40: 298-303, 2013.

37 U.S. National Library of Medicine: Topical or ablative treatment in preventing anal cancer in patients with HIV and anal high-grade squamous intraepithelial lesions [Internet]. ClinicalTrials.gov. Available from: https://clinicaltrials.gov/ct2/show/NCT02135419? term $=$ ANCHOR\&rank=8.

Received November 13, 2018

Revised November 27, 2018

Accepted November 29, 2018 